\title{
Multi-asset option pricing based on exponential Lévy process
}

\author{
Nan Liü, a, Meiling Wang ${ }^{1,2}$ and Xuebin Lü ${ }^{3}$ \\ ${ }^{1}$ the PLA Information Engineering University, Zhengzhou, China, 450001 \\ ${ }^{2}$ School of Telecommunication Engineering, Xidian University, Xi'an, China, 710071 \\ ${ }^{3}$ College of Science, Nanjing University of Technology, Nanjing, China, 211816 \\ aliunan526@126.com
}

Keywords: Multi-asset option pricing; Lévy process; Esscher transform.

\begin{abstract}
The multi-dimensional Esscher transform was used to find a locally equivalent martingale measure to price the options based on multi-asset. An integro-differential equation was driven for the prices of multi-asset options. The numerical method based on the Fourier transform was used to calculate some special multi-asset options in exponential Lévy models. As an example we give the calculation of extreme options.
\end{abstract}

\section{Introduction}

Lévy processes have been long used in mathematical finance to characterize stock-price model, the most famous model is Brownian motion. Black and Scholes derive an analytical expression of the European options based on the geometric Brownian motion, this model is well-known as Black-Scholes model. Recent years more generalized Lévy processes with jumps which can better fit the stock-price model have been introduced in financial models[1]. However, Lévy processes are jump processes which make the market incomplete, and there are many choices of equivalent martingale measure for risk-neutral derivative securities in exponential Lévy model[2]. One mathematically tractable choice is the so-called Esscher transform which was introduced to mathematical finance by Shiu[3] and Madan[4]. Raible[5] use this approach to price options in Generalized Hyperbolic Lévy model in one-dimensional situation. In the delight of the Esscher transform of one dimensional Lévy processes used by Raible to price options in Lévy model, we introduce Esscher transform of multi-dimensional Lévy processes to find the equivalent martingale measure. Under the equivalent martingale measure, we derive an integro-differential equation for the prices of multi-asset options. Moreover, we use the Fourier transform to get a numerical method to calculation some special multi-asset options in exponential Lévy models, as an example we give the calculation of extreme options.

\section{Market Model}

We consider a financial market model introduced in [6] consisting of a risk-less bond and several risky stocks which follow exponential Lévy processes. Assume that the market is no-arbitrage and has no transaction costs, and the stocks prices denoted by $\left(S_{i}(t)\right)_{t \geq 0}$ satisfy the following equations:

$$
S_{i}(t)=S_{i}(0) \exp \left(r t+\sum_{j=1}^{n} \sigma_{i j} L_{j}(t)\right), i=1,2, \cdots n
$$

Where $\mathrm{r}$ is the risk-less interest rate, and $(L(t))_{t \geq 0}=\left(L_{1}(t), \cdots, L_{n}(t)\right)^{T}{ }_{t \geq 0}$ is an n-dimensional Lévy process. We assume $L_{1}, \cdots, L_{n}$ to be mutually independent, i.e. $L_{1}, \cdots, L_{n}$ are all one-dimensional Lévy process and the probability that two component have one jump at the same time is zero[7]. $\sigma=\left(\sigma_{i j}\right)$ is an invertible matrix. Since the assets are on the same market, we use the linear combination of the same Lévy processes to show the dependence relation. This model is the 
generalization of the classical multi-asset Black-scholes model[8]. We denote the moment generating function of $L(1)$ by $m g f(u)=E(\exp \langle u, L(t)\rangle), u=\left(u_{1}, \cdots, u_{n}\right)^{T} \in R^{n}$, where naturally by the property of independent stationary increments of $L$ we get $m g f(u)^{t}=E(\exp \langle u, L(t)\rangle)$.

\section{Esscher Transform}

Risk-less martingale measure is the basis of pricing derivatives under the condition that the market is no-arbitrage. In the classical Black-scholes model the martingale conditions can uniquely determine the equivalent martingale measure to price options. However, if we introduces the general Lévy processes with jumps into the model, the martingale measure is not uniquely determined by only one condition measure[1]. There are many methods to find equivalent martingale measure. We introduce Esscher transform of multi-dimensional Lévy processes to find the equivalent martingale measure to price multi-assets options. First we give the definition of Esscher transform of multi-dimensional Lévy process.

Definition. Let $\mathrm{L}$ be a Lévy process on $R^{n}$ defined on the filtered probability space $\left(\Omega,\left(F_{t}\right)_{t \geq 0}, P, R^{n}\right)$, we call Esscher transform any change $\mathrm{P}$ to a locally equivalent measure $\mathrm{Q}$ with a density process $Z_{t}=\left.\frac{d Q}{d P}\right|_{F_{t}}$ of the expression:

$$
Z_{t}=\frac{\exp \langle\theta, L(t)\rangle}{m g f(\theta)^{t}}
$$

where $\theta=\left(\theta_{1}, \cdots, \theta_{n}\right)^{T} \in R^{n}$ with $\operatorname{mgf}(\theta)=E(\exp \langle\theta, L(1)\rangle)<\infty$.

Proposition. Eq.2 define a density proces for all $\theta=\left(\theta_{1}, \cdots, \theta_{n}\right)^{T} \in R^{n}$ with $m g f(\theta)=E(\exp \langle\theta, L(1)\rangle)<\infty$. L is a n-dimensional Lévy process under the new measure $Q$. Particularly, an n-dimensional Lévy process with independent component is again a Lévy process with independent components under the new measure $Q$.

Next we give the following assumption:

Assumption (1). The process $\mathrm{L}$ is non-degenerate Lévy process and possesses moment generating function $m g f: u \rightarrow E(\exp \langle u, L(1)\rangle)$ on interval $\prod_{i=1}^{n}\left(a_{i}, b_{i}\right)$, with $b_{i}-a_{i} \geq \max _{1 \leq i \leq n} \sigma_{i j}$, for $i=1,2, \cdots, n$.

Assumption (2). There exists a real vector $\theta=\left(\theta_{1}, \cdots, \theta_{n}\right)^{T}$ with $\theta_{i} \in\left(a_{i}, b_{i}\right), i=1,2, \cdots, n$ described in assumption (1) such that: $\operatorname{mgf}(\theta)=\operatorname{mgf}\left(\theta+\sigma_{i}\right)$, where $\sigma_{i}=\left(\sigma_{i 1}, \cdots, \sigma_{i n}\right)^{T}, i=1,2, \cdots, n$.

Theorem 1. Assume the stock prices processes are given by Eq.1 and assumption (1) (2) satisfied. The basic probability $\mathrm{P}$ is locally equivalent to a measure $Q$ such that the discounted prices of any stock $\exp (-r t) S_{i}(t)=S_{i}(0) \exp \left(\sum_{j=1}^{n} \sigma_{i j} L_{j}(t)\right)$ is a $Q$ martingale for $i=1,2, \cdots, n$. A density process leading to such a martingale measure $Q$ is given by the Esscher transform density:

$$
Z_{t}=\frac{\exp \langle\theta, L(t)\rangle}{m g f(\theta)^{t}}
$$

Where, $\theta$ satisfies: $\operatorname{mgf}(\theta)=m g f\left(\theta+\sigma_{i}\right), i=1,2, \cdots, n$

\section{Multi-assets Option Pricing by Esscher Transform}

The locally absolutely continuous measure transformation is important in mathematical finance which changes the underlying probability to risk-neutral measure. Under the risk-neutral measure all enterable discounted price processes are assumed to be martingales. Therefore such a measure is 
called martingale measure. By virtue of this assumption, prices of certain claims whose payoff at the strike date $T$ are known as functions of the underlying assets can be calculated for all the time $t \leq T$ by just taking the conditional expectation. For example, a European multi-asset option whose payoff at the strike date $T$ is a function of the underlying assets $S_{1}, S_{2}, \cdots, S_{n}$, that is $V_{T}=\omega\left(S_{1}(T), S_{2}(T), \cdots, S_{n}(T)\right)$, by the martingale assumption, is a martingale, that is $e^{-r t} V_{t}=E_{Q}\left(e^{-r T} V_{T} \mid F_{t}\right)$ for all $t \leq T$, then we can get the price of this option at time $0 \leq t \leq T$ :

$$
V_{t}=E^{Q}\left(e^{-r(T-t)} V_{T} \mid F_{t}\right)=e^{-r(T-t)} E^{Q}\left(\omega\left(S_{1}(T), S_{2}(T), \cdots, S_{n}(T)\right) \mid F_{t}\right)
$$

Especially, the price of this option at the initial time is:

$$
V=E^{Q}\left(e^{-r T} V_{T}\right)=e^{-r T} E^{Q}\left(\omega\left(S_{1}(T), S_{2}(T), \cdots, S_{n}(T)\right)\right)
$$

From the property of independent and stationary increments of the Lévy process, we get:

$$
V_{t}=e^{-r(T-t)} E^{Q}\left(\left.\omega\left(y_{1} e^{r(T-t)+\sigma_{1} L_{T-t}}, \cdots, y_{n}(t) e^{\left.r(T-t)+\sigma_{n} L_{T-t}\right)}\right)\right|_{y_{1}(t)=S_{1}(t), \cdots, y_{n}(t)=S_{n}(t)}\right.
$$

So the price of the option is a function of the present prices of underlying assets, that is, it is only dependent on the time $\mathrm{t}$ and the present prices of underlying assets. Then we can denote $V_{t}=g\left(S_{1}(t), S_{2}(t), \cdots, S_{n}(t), t\right)$, with

$$
g\left(y_{1}, \cdots, y_{n}, t\right)=e^{-r(T-t)} E^{Q}\left(\omega\left(y_{1} e^{r(T-t)+\sigma_{1} L_{T-t}}, \cdots, y_{n}(t) e^{\left.r(T-t)+\sigma_{n} L_{T-t}\right)}\right)\right.
$$

Let $X_{i}(t)=\log e^{r(T-t)} S_{i}(t), i=1,2, \cdots, n$, then we get:

$$
\begin{aligned}
& V_{t}=f\left(X_{1}(t), \cdots, X_{n}(t), t\right) \\
& =e^{-r(T-t)} E_{Q}\left(\left.\omega\left(e^{x_{1}+\sigma_{1} L_{T-t}}, \cdots, e^{\left.x_{n}(t)+\sigma_{n} L_{T-t}\right)}\right)\right|_{\chi_{1}(t)=X_{1}(t), \cdots, x_{n}(t)=X_{n}(t)}\right.
\end{aligned}
$$

Then we can derive an integro-differential equation of the multi-asset option prices:

Theorem 2. Assume that the function defined by Eq.8 is twice continuously differentiable with respect to $x_{i}$ and is once continuously differentiable with respect to t, the support of $L_{i}$ is $\mathrm{R}$, then $f\left(x_{1}, \cdots, x_{n}, t\right)$ satisfies the following equation:

$$
\begin{aligned}
& -r f\left(x_{1}, \cdots, x_{n}, t\right)+\partial_{t} f\left(x_{1}, \cdots, x_{n}, t\right)+\sum_{i, j=1}^{n} \sigma_{i j} b_{j} \partial_{i} f\left(x_{1}, \cdots, x_{n}, t\right)+ \\
& \frac{1}{2} \sum_{i, j, k=1}^{n} \sigma_{i k} \sigma_{j k} c_{k} \partial_{i} \partial_{j} f\left(x_{1}, \cdots, x_{n}, t\right)+\int_{R^{n}}\left\{f\left(x_{1}+\sum_{j=1}^{n} \sigma_{1 j} y_{j}, \cdots, x_{n}+\sum_{j=1}^{n} \sigma_{n j} y_{j}, t\right)\right. \\
& \left.-f\left(x_{1}, \cdots, x_{n}, t\right)-\sum_{i, j=1}^{n} \sigma_{i j} y_{j} \partial_{i} f\left(x_{1}, \cdots, x_{n}, t\right)\right\} v^{L}\left(d y_{1}, \cdots, d y_{n}\right)=0
\end{aligned}
$$

where $b=\left(b_{1}, \cdots, b_{n}\right)^{T} \in R^{n}, c=\operatorname{diag}\left(c_{1}, \cdots, c_{n}\right)$ with $c_{i} \geq 0, v^{L}$ is the Lévy measure of L under the measure $Q,(b, c, v)$ is the Lévy-Khintchine generating triplet of $\mathrm{L}$ under the measure $Q$.

From Theorem 2 we can derive an integro-differential equation of the multi-asset option prices, now we give a convenient numerical method to calculate some special multi-asset option. Use Laplace transform of multi-variable functions we have the following theorem and give a convenient method to calculate some special multi-asset option:

Let $\zeta_{i}=-\ln \left(S_{i}(0) e^{r T}\right), X_{i}=-\sigma_{i}^{T} L, i=1,2, \cdots, n$, then terminal payoff of a given multi-asset option is:

$$
V_{T}=\omega\left(S_{1}(T), S_{2}(T), \cdots, S_{n}(T)\right)=\omega\left(e^{\zeta_{1}-X_{1}(T)}, \cdots, e^{\zeta_{n}-X_{n}(T)}\right) \triangleq v(\zeta-X(T))
$$


Theorem 3. Given a multi-asset option with terminal payoff $\omega\left(S_{1}(T), S_{2}(T), \cdots, S_{n}(T)\right)$, let $v(x)=\omega\left(e^{-x_{1}}, \cdots, e^{-x_{n}}\right), \quad x=\left(x_{1}, \cdots, x_{n}\right)^{T} \in R^{n}, x \rightarrow e^{-R^{T} x}|v(x)|$ is bounded and integral for some $R=\left(R_{1}, \cdots, R_{n}\right)^{T} \in R^{n}$ such that the moment generating function of $X_{T}$ exist .

Then the price of this option at time 0 is:

$$
V(\xi)=\frac{1}{(2 \pi)^{n}} e^{\langle\xi, R\rangle-r T} \int_{-\infty}^{-\infty} \cdots \int_{-\infty}^{+\infty} e^{i\langle\xi, u\rangle} L[v](R+i u) L[\rho](R+i u) d u
$$

where $L[\rho](R+i u)=\kappa(i R-u), \kappa(z)=m g f\left(\sigma^{T} z+\theta\right) / m g f(\theta)$, and the integral exists as the limit $\lim _{M_{1} \rightarrow \infty} \cdots \lim _{M_{n} \rightarrow \infty} \int_{-M_{1}}^{M_{1}} \cdots \int_{-M_{1}}^{M_{1}} e^{i\langle\xi, u\rangle} L[v](R+i u) L[\rho](R+i u) d u_{1} \cdots d u_{n}$.

\section{Summary}

With the model and method introduced in this paper, we can calculate the price of multi-asset options which follow exponential Lévy processes.Use theorem 3, we can calculate some special multi-asset option numerically. For example, we consider the pricing of options on the extreme of several risky assets. The European put and call options on the extreme of several risky assets are applicable in a wide variety of contingent claims in corporate finance and bond pricing model. The terminal payoff of the European put and call options on the minimum and maximum of several risky assets $S_{1}, \cdots S_{n}$ are respectively: $C_{\max }=\left(\max \left(S_{1}, \cdots S_{n}\right)-K\right)^{+}, C_{\min }=\left(\min \left(S_{1}, \cdots S_{n}\right)-K\right)^{+}$, where $(x)^{+}=\max (x, 0)$.

\section{References}

[1] D. Applebaum, Lévy processes and stochastic calculus, Cambridge University Press, Cambridge, 2004.

[2] E. Eberlein, J. Jacod, On the range of option prices, Finance and Stochastics, 1 (1997) 131-140.

[3] H.U. Geber, E.S.W. Shiu, Actuarial bridges to dynamic hedging and option pricing, Mathematics and Economics, 18 (1996) 183-218.

[4] D.B. Madan, F. Milne, Option pricing with VG martingale components, Math Finance, 1 (1991) 39-55.

[5] S. Raible, Lévy processes in finance, theory, numerics and empirical facts, Ph. D Theis, University of Freiberg, 2000.

[6] S. Emmer, C. Klüpplebberg, Optimal portfolio when stock prices follow exponential Lévy processes, Math Finance, 8 (2004) 17-44.

[7] K. Sato, Lévy processes and infinitely divisible distributions, Cambridge University Press, Cambridge, 1999.

[8] Y.K. Kwok, Mathematical models of financial derivatives, Springer, 1998. 